(1)

George Fox

UNIVERSITY
Digital Commons @ George Fox University

Faculty Publications - School of Physical

Therapy

2020

Overall Health and the Influence of Physical Therapy on Physical

Function Following Total Ankle Arthroplasty

Frank E. DiLiberto

Daniel H. Aslan

Jeff R. Houck

Bryant S. Ho

Anand M. Vora

See next page for additional authors

Follow this and additional works at: https://digitalcommons.georgefox.edu/pt_fac

Part of the Physical Therapy Commons 
Authors

Frank E. DiLiberto, Daniel H. Aslan, Jeff R. Houck, Bryant S. Ho, Anand M. Vora, and Steven L. Haddad 


\title{
Overall Health and the Influence of Physical Therapy on Physical Function Following Total Ankle Arthroplasty
}

\author{
Frank E. DiLiberto, PT, PhD' , Daniel H. Aslan, MS², Jeff R. Houck, PT, PhD², \\ Bryant S. Ho, MD 4 , Anand M. Vora, MD5, and Steven L. Haddad, MD ${ }^{6}$
}

\begin{abstract}
Background: The overall health and the importance of physical therapy for people following total ankle arthroplasty (TAA) have been understudied. Our purpose was to characterize the overall health of patients following TAA, and explore the frequency, influence, and patient-perceived value of physical therapy.

Methods: People who received a TAA participated in this retrospective cohort online survey study. The survey included medical history questions and items from the Patient-Reported Outcomes Measurement Information System (PROMIS) Short Forms. Seven PROMIS domains, reflecting the biopsychosocial model of care (physical, mental, social), were included to examine participant overall health status in comparison to the general population. Items regarding physical therapy participation (yes/no), number of visits, and perceived value (scale 0-10; $10=$ extremely helpful) were also included. Descriptive statistics were generated for participant characteristics, PROMIS domain $T$ scores, and physical therapy questions. The influence of participant characteristics or physical therapy visits on PROMIS domain $T$ scores that scored below the population mean were examined with multiple linear regression or ordinal regression.

Results: The response rate was $61 \%(n=95)$. Average postoperative time was approximately 3 years (mean [SD]: 40.0 [35.3] months). Physical function and ability to participate in social roles and activities domain $T$ scores were at least I SD below the population mean. Most patients received physical therapy (86\%; I7.I [ I I.0] visits) and found it helpful (7.2 [3.0]). Participant characteristics were minimally predictive of physical function and social participation $T$ scores. Number of physical therapy visits predicted physical function $T$ scores $(P=.03)$.

Conclusions: Most health domain scores approached the population mean. Physical therapy was perceived to have a high value, and greater visits were related to greater physical function. However, lower physical function and social participation scores suggest that postoperative care directed toward these domains could improve the value of TAA and promote overall health.
\end{abstract}

Level of Evidence: Level III, retrospective comparative study.

Keywords: total ankle arthroplasty, overall health, physical therapy, physical function, PROMIS

\section{Introduction}

The application of total ankle arthroplasty (TAA) to treat end-stage ankle arthritis is steadily increasing. ${ }^{20,29,31}$ Increased use of TAA can be partially attributed to improved implant survivorship, as well as improving complication and revision rates. ${ }^{16,18,21,25}$ Additional outcomes such as high patient satisfaction, pain reduction, preservation and improvement of ankle motion, lowered risk of adjacent joint arthritis (vs ankle arthrodesis), and improved scores on foot-specific functional scales also support the use of TAA in the treatment of end-stage ankle arthritis. ${ }^{8,11,18,28}$

Although TAA outcomes are positive, measures of overall health following TAA have been underused. Examination
'Department of Physical Therapy, Physical Therapy Movement Analysis Laboratory, College of Health Professions, Rosalind Franklin University of Medicine \& Science, North Chicago, IL, USA

${ }^{2}$ Department of Kinesiology, College of Applied Health Sciences, University of Illinois at Urbana-Champaign, Champaign, IL, USA

${ }^{3}$ School of Physical Therapy, George Fox University, Newberg, OR, USA

${ }^{4}$ Hinsdale Orthopaedics, A Division of Illinois Bone \& Joint Institute, Hinsdale, IL, USA

${ }^{5}$ Illinois Bone \& Joint Institute, Libertyville, IL, USA

${ }^{6}$ Illinois Bone \& Joint Institute, Glenview, IL, USA

Corresponding Author:

Frank E. DiLiberto, PT, PhD, Department of Physical Therapy, Physical therapy Movement Analysis Laboratory, College of Health Professions, Rosalind Franklin University of Medicine \& Science, 3333 Green Bay Road, North Chicago, IL 60064, USA.

Email: frank.diliberto@rosalindfranklin.edu 
of multiple domains of health to characterize overall health is increasingly recognized as an important aspect of patient reported outcomes and healthcare, including foot and ankle care. ${ }^{5,15}$ Current information regarding patient reported outcomes following TAA are dominated by region specific / disease specific foot and ankle scales, and less commonly, the physical or mental subsets of generic patient reported outcomes. ${ }^{11,18,28}$ Relatively few studies have considered overall health or multiple domains of health..$^{23,24,30,34,35}$ One option to efficiently characterize patient health status is the PatientReported Measurement Information System (PROMIS). In reflection of the biopsychosocial model of care, PROMIS outcome measures include multiple domains of health to provide person-centered assessments of function (physical), symptoms (psychological), and social impact, which can be compared to population mean values. ${ }^{5,36}$ Information gained from PROMIS scales are also clinically translatable because patient scores can be used to guide postoperative care in the form of secondary provider referrals (ie, referral to sleep medicine) and/or direct patient-provider interactions. For example, if a domain such as the ability to participate in social roles is lower than expected following TAA, patientprovider consultation may help guide exercises to directly enhance a patient's ability to participate in a given social activity. In this way, assessment of multiple domains of health can foster comprehensive patient care, and in the case of TAA, improve the patient perceived value of the procedure.

While postoperative care and rehabilitation likely contribute to TAA treatment success and patient perceived value, literature regarding postoperative care, and specifically physical therapy (PT), is extremely limited. In contrast, there is abundant literature regarding the use and importance of PT for other arthroplasty procedures (ie, hip, knee, shoulder). ${ }^{4,22}$ Currently, TAA postoperative literature is focused on peri-operative interventions, as opposed to rehabilitation interventions. Few studies have reported on the frequency of rehabilitation or PT, or provided discussion regarding rehabilitation approaches following TAA.,17,19,30 Accordingly, the frequency, patient perceived value, and potential influence PT has on patient-reported outcomes is unexplored. However, obtaining a profile of PT use and value is the first step to developing and modifying current rehabilitation approaches to leverage TAA operative success.

The purpose of this study was to characterize the overall health of patients following TAA, and to explore the frequency, influence, and patient perceived value of PT. We hypothesized that PROMIS scores would fall within 1 SD of the population mean across all health domains, and that select patient characteristics (ie, age) would predict variance in PROMIS scores. Although exploratory, we also hypothesized that most patients would receive PT and perceive PT as helpful, and that greater PT use would be associated with greater physical function scores.

\section{Methods}

This was a retrospective cohort single time-point online survey study of individuals who had received a TAA at least 6 months previously. Participants were recruited from large social media platforms (approximately 3000 possible individuals), and from participants of related laboratory studies. The study was approved by the Institutional Review Board and all subjects provided informed consent.

The 58-item survey was administered via a university supported online platform (Qualtrics, Provo, UT). A link to the survey was posted on social media sites at 2-week increments over the course of 2 months. Emails with a survey link were sent to individuals who were recruited from related laboratory studies. The survey was open for 2 months (February to April 2019). Per our ethical requirements, participants were allowed to skip questions if they so desired. Participant compensation was not offered.

Medical history questions pertaining to age, gender, body mass index (BMI), postoperative time since surgery, complication history, and comorbidities were recorded. BMI was calculated from participant-reported height and weight estimates. Time since surgery was measured in months. Participants were asked if they had a complication with TAA, and if yes, were asked to describe the complication (write in response). Participant self-reported descriptions of perceived complications were independently reviewed by 2 fellowship-trained foot and ankle orthopedic surgeons with extensive experience in TAA. These descriptions were categorized as a true intraoperative or postoperative complication associated with TAA, or an expected potential issue or unrelated issue following TAA. Descriptions clearly reflecting low-, medium-, and high-grade complications were treated as a yes response. ${ }^{1,9}$ Participant descriptions not fitting these criteria were counted as a no response along with those who initially responded no to our complications question. Discrepancies between surgeon categorizations of participant descriptions were settled by a third independent fellowship-trained foot and ankle orthopedic surgeon. Our approach resulted in 26 surgeon-ratified complications out of a possible 41 participant self-reported complication descriptions (Supplemental File S1). Participants were also asked if they ever had 1 of the following comorbidities: high blood pressure, cardiac conditions (heart attack, congestive heart failure, chest pain), pulmonary conditions (emphysema, chronic obstructive pulmonary disease, asthma), neurologic conditions (Parkinson, multiple sclerosis), cancer, stoke, and other (write in response). Responses were categorized as having zero or at least 1 comorbidity. These participant characteristics were selected because of their potential influence on patient-reported outcomes. ${ }^{6,33}$

Items from the PROMIS-29 Short Form (v2.1) were included in the online survey. The PROMIS-29 includes 4 items for 7 domains of health (Figure 1) and an additional 


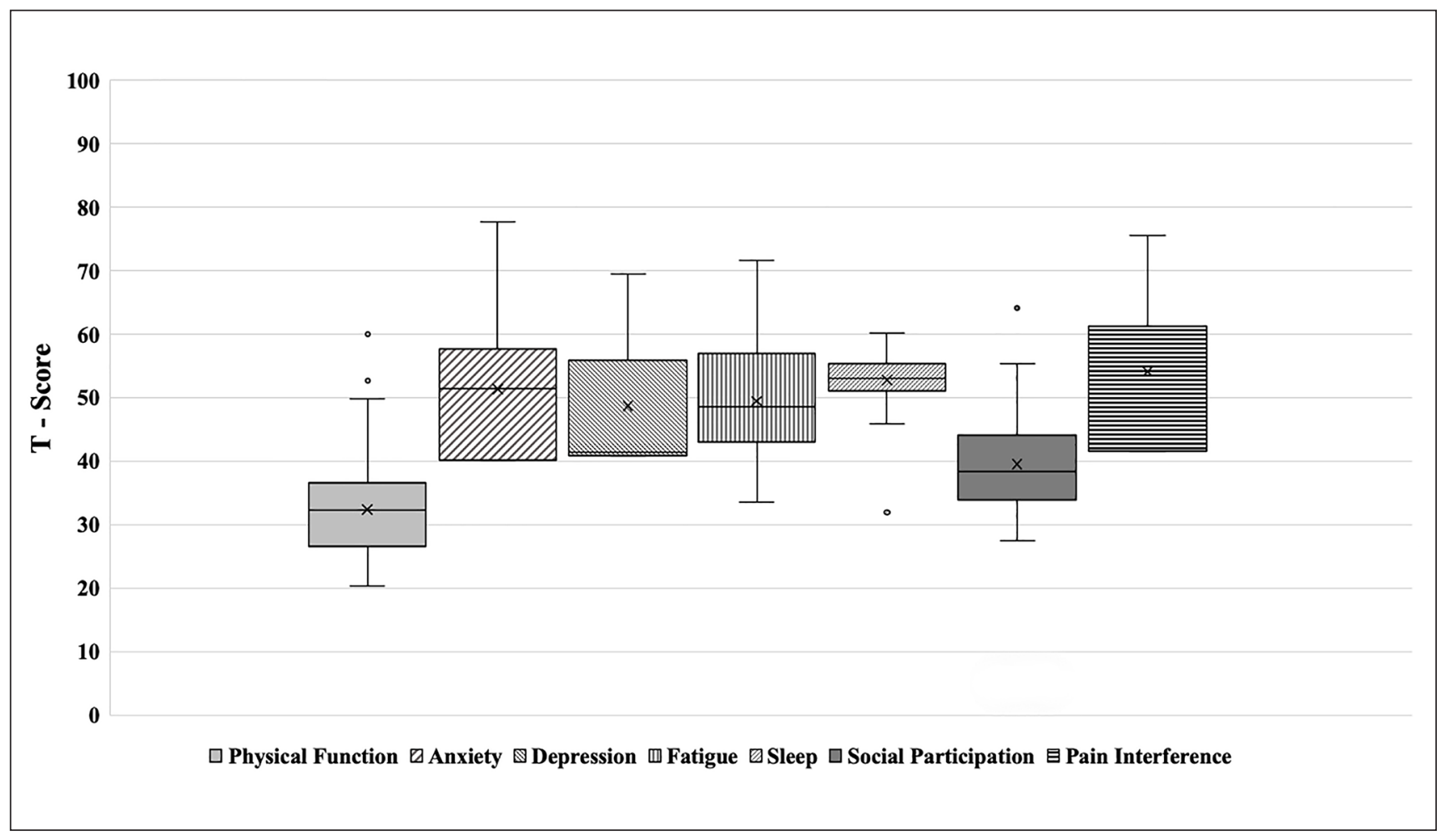

Figure I. PROMIS domain $T$ scores. Standard box plots along with an $x$ to indicate mean values are presented. Scores of 50 represent the population mean with \pm 10 equaling I SD.

item regarding pain intensity, which was not included in our analysis. The PROMIS-29 is reliable and valid, and use of PROMIS is recommended in people following foot and ankle surgery. ${ }^{5,12,14,15}$ We were also particularly interested in domains of health that we expected to be related to physical therapy. Therefore, we included an additional four items for physical function, which, when combined with the four physical function items of the PROMIS-29 scale, resulted in 8 total physical function items and represented the PROMIS Physical Function-8b Short Form (v2.0). PROMIS $T$ scores were calculated for each domain, for each participant, in accordance with instrument guidelines. $T$ scores of 50 represent population means with an SD of \pm 10 equating to $1 \mathrm{SD}$. Access to PROMIS scales, scoring methodology, and interpretation of scores is freely available online (healthmeasures.net).

Three main PT-related questions were also included in the survey. Participants were asked if they had formal PT following TAA (yes/no). They were also asked to estimate the total number of PT visits on a scale that ranged from zero to 30 or more. Zero was used for this question if a participant did not receive PT. Third, participants were asked to rate if PT was helpful on a $0-10$ scale, with zero being not helpful to 10 being extremely helpful.

\section{Statistical Analysis}

An a priori power analysis was conducted. A sample of 92 was required to achieve a moderate effect for our planned multiple linear regression analyses between 6 participant characteristics and $T$ scores $\left(f^{2}=0.16, \alpha=0.05,1-\beta=0.80\right)$.

A survey was considered complete if a participant responded to $95 \%$ of the items. The response rate was calculated as (number of completed surveys / number of people who accessed the survey) $\times 100$. Missing data were addressed with a mean imputation method with random variability that was based on the present distribution. This approach reduces bias in parameter estimates by representing the expected variance of a given variable based on the sample data. ${ }^{10,32}$ Data were inspected for normality and variance homogeneity. Postoperative time $(1.7,3.3 ; P<$ $.01)$, number of PT visits $(-0.11,-1.43 ; P<.01)$, and perceived helpfulness of PT $(-1.0,0.1 ; P<.01)$ were not normally distributed (skewness, kurtosis, and Shapiro-Wilk test). Postoperative time and perceived helpfulness of PT data were not altered. PT visits were categorized into no PT (zero visits; $12.6 \%$ of the sample), some PT (1-29 visits; $56.8 \%$ ), and most PT (30 or more visits; $30.5 \%$ ) based on the distribution of the sample data. PROMIS $T$ scores were 
Table I. Participant characteristics $(\mathrm{N}=95)$.

\begin{tabular}{lccr}
\hline & Mean (SD) or \% & Median & Range $^{\mathrm{a}}$ \\
\hline Age, y & $59.2($ II.5) & 60 & $26-82$ \\
Gender, \% female & 62 & & \\
Body mass index & $28.9(5.7)$ & 27.7 & $19.1-44.3$ \\
Postoperative time, mo & $40.0(35.4)$ & 27 & $6-174$ \\
Complications, \% yes & 27 & & \\
Comorbidities, \% yes & 58 & & \\
\hline
\end{tabular}

aRange is presented as minimum value to maximum value.

Table 2. Physical therapy (PT) questions.

\begin{tabular}{lcrr}
\hline & \% or Mean (SD) & Median & Range $^{\mathrm{a}}$ \\
\hline PT participation, \% yes & 87 & & \\
PT visits (total number) & $17.1(\mathrm{II.0})$ & 15 & $0-30$ \\
Perceived helpfulness of PT & $7.2(3.0)$ & 8 & $0-10$ \\
$\quad(0-10$ scale) & & & \\
\hline
\end{tabular}

aRange is presented as minimum value to maximum value.

also not normally distributed; these data were not altered. Descriptive statistics were used to evaluate participant characteristics, PROMIS domain $T$ scores, and PT-specific questions. Separate multiple linear regressions with bootstrapping, to address non-normal data distributions, between participant characteristics, and PROMIS domain $T$ scores that were at least $1 \mathrm{SD}$ below the population mean were conducted. ${ }^{37}$ Ordinal regression was conducted between categorized PT visits and the same PROMIS domain $T$ scores, and the test of parallel lines was used to evaluate PT visit categorization. An a priori alpha of 0.05 was selected for all analyses.

\section{Results}

The response rate of $61.2 \%(95 / 155)$ resulted in a sample of 95 people. The sample consisted of more females than males and an approximate mean age of 60 years old (Table 1). Most PROMIS domain $T$ scores approached the population mean of 50. However, physical function (mean 32.4 [SD 7.8], 95\% CI 30.8-34.0; median 32.3) and ability to participate in social roles and activities (social participation) (39.6 [9.0], 37.7-41.4; 38.5) $T$ scores fell at least $1 \mathrm{SD}$ below the population mean (Figure 1). Eighty-seven percent of individuals received formal PT for an estimated 17.1 visits per person (Table 2). The mean perceived helpfulness of PT was 7.2/10. Physical function and social participation $T$ scores were largest in the most PT visit category (Figure 2).

Participant characteristics accounted for $15 \%(P=.04)$ and $7 \%(P=.42)$ of the variance in physical function and social participation $T$ scores, respectively (Table 3 ). Increasing age was significantly associated with lower social participation $T$ scores. Physical function $T$ scores predicted
PT visit category $(P=.03)$, but social participation scores did not (Table 4). Test of parallel lines for both ordinal regressions were not statistically significant, supporting our categorization of PT visits.

\section{Discussion}

Our study is one of a few studies to examine multiple domains of health, and the first study to examine the role of PT, in people following TAA. Key findings included low physical function and social participation scores, which were marginally related to common patient characteristics anticipated to influence outcomes. These findings indicated that participants' perception of their ability to perform certain physical activities or participate in desired social activities were lower than the general population and not substantially dependent on factors such as postoperative time, comorbidities, or BMI. Additionally, most participants received several PT sessions and found it helpful. Higher physical function scores were found in those with a greater number of PT visits. Accordingly, study findings raise concern regarding physical function and social participation following TAA and suggest that while PT appears utilized and valuable, additional strategies are needed to improve patient physical function.

At an average of approximately three years following TAA, surveyed patients scored near the population mean on most of the examined health domains (Figure 1). Unique to the present study was the assessment of sleep quality, fatigue, and anxiety in people following TAA. Patients scored within $1 \mathrm{SD}$ of the population mean in these domains (Figure 1). Recent reports on depression in people 6 months following TAA suggest depression is slightly improved from preoperative scores, and within normal limits following TAA. ${ }^{24,30}$ Similarly, pain and pain interference scores are improved and approach normal levels 6-24 months following TAA..$^{23,24,30}$ The present study results suggest normative levels of depression and pain interference can be expected longer-term. Overall, patient scores that were close to the population mean on the majority of examined health domains adds to the already positive literature regarding TAA outcomes.

Physical function and social participation were the main domains of concern because patients scored at least $1 \mathrm{SD}$ below the population mean. Although not fully analogous, McConnell and Queen ${ }^{23}$ reported physical function SF-36 scores lower than age-matched normative scores in people 2 years following TAA. ${ }^{13}$ Social role scores were also slightly lower than normal levels. The present study mirrors this study in that physical function was lower than social participation $T$ scores, and both domains were lower than desired, even at a mean postoperative time of three years (Figure 1). Accordingly, it appears that physical function likely plateaus at postoperative times beyond the 


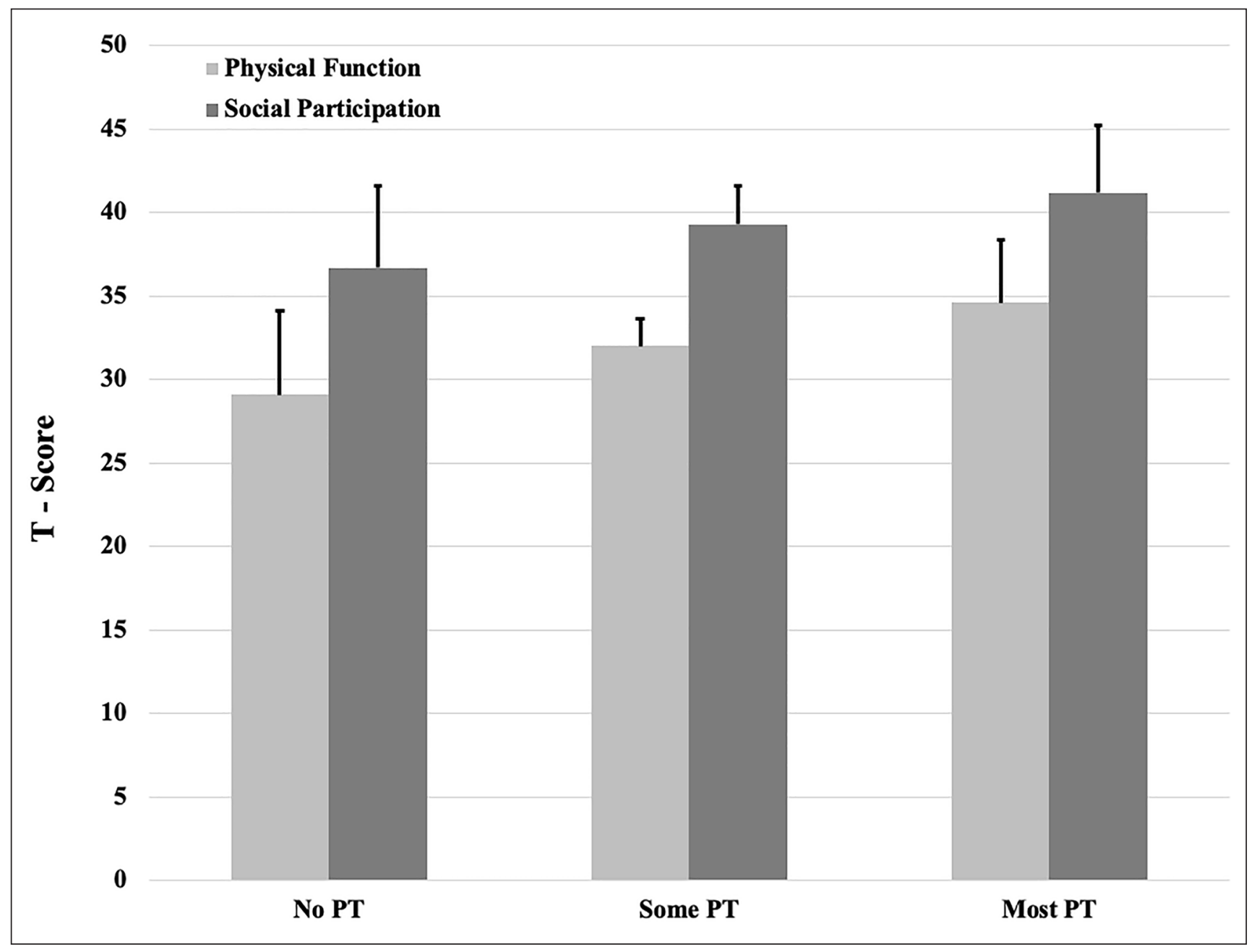

Figure 2. Cohort mean PROMIS T scores categorized by number of physical therapy (PT) visits. Physical function and social participation domains are presented. Error bars correspond to the $95 \%$ confidence limits.

Table 3. Multiple Linear Regression Between Participant Characteristics and Select PROMIS T Scores. ${ }^{a}$

\begin{tabular}{|c|c|c|c|c|}
\hline & \multicolumn{2}{|c|}{$\begin{array}{c}\text { Physical function } \\
\left.\text { (Model } R^{2}=0.14 ; P=.04 *\right)\end{array}$} & \multicolumn{2}{|c|}{$\begin{array}{c}\text { Social participation } \\
\left.\text { (Model } R^{2}=0.07 ; P=.34\right)\end{array}$} \\
\hline & $\begin{array}{c}B(\beta) \\
{[95 \% \mathrm{Cl}]}\end{array}$ & $P$ value & $\begin{array}{c}B(\beta) \\
{[95 \% \mathrm{Cl}]}\end{array}$ & $P$ value \\
\hline Age & $\begin{array}{l}-0.10(-0.15) \\
{[-0.24,0.03]}\end{array}$ & .13 & $\begin{array}{c}-0.21(-0.26) \\
{[-0.37,-0.06]}\end{array}$ & $.01 *$ \\
\hline Gender & $\begin{array}{c}2.52(0.16) \\
{[-1.08,5.90]}\end{array}$ & .16 & $\begin{array}{c}1.03(0.06) \\
{[-3.03,5.03]}\end{array}$ & .62 \\
\hline Body mass index & $\begin{array}{c}0.20(0.15) \\
{[-0.07,0.47]}\end{array}$ & .13 & $\begin{array}{l}-0.01(-0.01) \\
{[-0.38,0.31]}\end{array}$ & .96 \\
\hline Postoperative time & $\begin{array}{c}0.03(0.13) \\
{[-0.02,0.08]}\end{array}$ & .24 & $\begin{array}{l}-0.01(-0.02) \\
{[-0.06,0.04]}\end{array}$ & .81 \\
\hline Complications & $\begin{array}{c}2.48(0.15) \\
{[-0.34,5.47]}\end{array}$ & .10 & $\begin{array}{c}1.67(0.08) \\
{[-2.46,5.91]}\end{array}$ & .44 \\
\hline Comorbidities & $\begin{array}{c}2.04(0.13) \\
{[-1.24,5.30]}\end{array}$ & .22 & $\begin{array}{c}0.75(0.04) \\
{[-3.17,4.81]}\end{array}$ & .72 \\
\hline
\end{tabular}

a Unstandardized coefficients ( $(B$ 's), confidence intervals $(C l s)$, and $P$ values are bootstrapped estimates. Standardized coefficients $(\beta)$ assume data normality. *Indicates statistical significance at $\alpha<.05$. 
Table 4. Ordinal Regression Between PT Visits (None, Some, Most) and Select PROMIS T Scores.

\begin{tabular}{|c|c|c|c|}
\hline & $\begin{array}{l}\text { Model Fit, } \\
\chi^{2}(P \text { Value })\end{array}$ & $\begin{array}{l}\text { Parameter estimate, } \\
\qquad[95 \% \mathrm{Cl}]\end{array}$ & $\begin{array}{c}\text { Test of parallel lines, } \\
P \text { value }\end{array}$ \\
\hline Physical function & $5.02(.03)^{*}$ & $\begin{array}{c}0.06 \\
{[0.01,0.1 \mathrm{I}]}\end{array}$ & .50 \\
\hline Social participation & $2.24(.14)$ & $\begin{array}{c}0.03 \\
{[-0.01,0.08]}\end{array}$ & .64 \\
\hline
\end{tabular}

Abbreviations: PROMIS, Patient-Reported Measurement Information System; PT, physical therapy.

*Indicates statistical significance at $\alpha<.05$.

1-2 years in which improvements from preoperative scores are notable. ${ }^{1,26,27,34}$

We suspect physical function is important for social participation and related to postoperative care. A post hoc analysis resulted in a significant correlation in $T$ scores between these 2 health domains $(\rho=0.77 ; P<.01)$. Further, our model of participant characteristics, while significant in total or in part, was actually minimally predictive of physical function or social participation $T$ scores (model $R^{2} \leq$ 0.15 ) (Table 3). One possible reason is that postoperative care, as opposed to patient characteristics, may be the main influential factor in achieving normal levels of physical function and consequent social participation. Therefore, we suggest postoperative care directed at physical function that will directly improve the ability to participate in social roles will benefit patients, promote overall health, and potentially prevent future health problems.

PT may be an important aspect of improving physical function following TAA. Our study findings demonstrate that PT following TAA is common, perceived as helpful, and associated with greater patient physical function (Table 2 and 4). In contrast, prior investigations have reported that referral to nursing or rehabilitation facilities following TAA approached $20 \%$, and that PT use following TAA approached $10 \% .{ }^{3,30}$ However, Rajapakshe et al ${ }^{30}$ acknowledged that PT use following TAA in their study was likely underestimated. To this point, nearly $90 \%$ of our sample received several PT visits (Table 2). Further, patients categorized as receiving the most PT ( $\geq 30$ visits) scored more than 5 points better on physical function than the patients who did not receive PT (Figure 2); exceeding the minimum clinically important difference in physical function for foot and ankle patients. ${ }^{12}$ The importance of physical function is underscored by the knowledge that PROMIS physical function scores can predict patient perceptions of surgical success or failure. ${ }^{2}$ Therefore, $\mathrm{PT}$ is both a relevant and important consideration when evaluating the influence of postoperative care on TAA treatment success, and particularly physical function.

Weaknesses of this study include the retrospective design, sampling strategy, and potential recall bias of participant responses related to PT questions. Although we suspect outcomes are improved following TAA, ${ }^{11,23,28}$ our study was not designed to evaluate change in outcome scores. It is also possible that only a subset of patients are represented in our sample (ie, individuals having online access or engaged in social media) or that our response rate was overestimated. We did not have a method to track how many people actually viewed the social media posts. Additionally, given the relatively wide range of postoperative times (6-174 months) participant recall bias may have influenced PT visit and perceived value of PT results. We did not have a way to verify PT visits. Although our categorization of PT visits partially guards against recall bias, alternate approaches may provide additional insight regarding the relationship between PT and physical function. In particular, our approach did not allow us to examine the quality, type, or rationale for PT. However, our sample is large, representative of participant characteristics commonly found in TAA literature, ${ }^{7,23}$ and offers a longer-term profile of multiple health domains in people following TAA. In total, study findings suggest the need for even larger (ie, multisite) prospective studies to evaluate multiple domains of health and the potential role PT has in achieving these outcomes. The strengths of this study include a relatively large sample, use of a validated measure to consider multiple domains of health, and a first look at PT use, frequency, and perceived value.

In conclusion, PT use is common, perceived to be valuable, and was related to greater physical function in patients following TAA. Lower PROMIS domain scores in physical function and social participation suggest that postoperative care directed at these domains will increase the value of TAA, postoperative care, and PT.

\section{Declaration of Conflicting Interests}

The author(s) declared no potential conflicts of interest with respect to the research, authorship, and/or publication of this article. ICMJE forms for all authors are available online.

\section{Funding}

The author(s) received no financial support for the research, authorship, and/or publication of this article.

\section{ORCID iD}

Frank E. DiLiberto, PT, PhD, $-5551-9481$

https://orcid.org/0000-0001

\section{Supplemental Material}

Supplementary material is available online with this article. 


\section{References}

1. Adams SBJ, Demetracopoulos CA, Queen RM, Easley ME, DeOrio JK, Nunley JA. Early to mid-term results of fixedbearing total ankle arthroplasty with a modular intramedullary tibial component. J Bone Joint Surg Am. 2014;96(23): 1983-1989.

2. Anderson MR, Baumhauer JF, DiGiovanni BF, et al. Determining success or failure after foot and ankle surgery using Patient Acceptable Symptom State (PASS) and Patient Reported Outcome Information System (PROMIS). Foot Ankle Int. 2018;39(8):894-902.

3. Boylan MR, Riesgo AM, Paulino CB, Sheskier SC. Does total ankle arthroplasty belong in the comprehensive care for joint replacement? J Foot Ankle Surg. 2018;57(1):69-73.

4. Bullock GS, Garrigues GE, Ledbetter L, Kennedy J. A systematic review of proposed rehabilitation guidelines following anatomic and reverse shoulder arthroplasty. J Orthop Sports Phys Ther. 2019;49(5):337-346.

5. Cella D, Riley W, Stone A, et al. The Patient-Reported Outcomes Measurement Information System (PROMIS) developed and tested its first wave of adult self-reported health outcome item banks: 2005-2008. J Clin Epidemiol 2010;63(11):1179-1194.

6. Cunningham DJ, DeOrio JK, Nunley JA, Easley ME, Adams $\mathrm{SB}$. The effect of patient characteristics on 1 to 2 -year and minimum 5-year outcomes after total ankle arthroplasty. $J$ Bone Joint Surg Am. 2019;101(3):199-208.

7. Daniels TR, Younger AS, Penner M, et al. Intermediateterm results of total ankle replacement and ankle arthrodesis: a COFAS multicenter study. J Bone Joint Surg Am. 2014;96(2):135-142.

8. Dekker TJ, Walton D, Vinson EN, et al. Hindfoot arthritis progression and arthrodesis risk after total ankle replacement. Foot Ankle Int. 2017;38(11):1183-1187.

9. Glazebrook MA, Arsenault K, Dunbar M. Evidence-based classification of complications in total ankle arthroplasty. Foot Ankle Int. 2009;30(10):945-949.

10. Graham JW. Missing data analysis: making it work in the real world. Annu Rev Psychol. 2009;60:549-576.

11. Hendy BA, McDonald EL, Nicholson K, et al. Improvement of outcomes during the first two years following total ankle arthroplasty. J Bone Joint Surg Am. 2018;100(17):1473-1481.

12. Ho B, Houck JR, Flemister AS, et al. Preoperative PROMIS scores predict postoperative success in foot and ankle patients. Foot Ankle Int. 2016;37(9):911-918.

13. Hopman WM, Towheed T, Anastassiades T, et al. Canadian normative data for the SF-36 Health Survey. Canadian Multicentre Osteoporosis Study Research Group. CMAJ. 2000;163(3):265-271.

14. Hung M, Baumhauer JF, Latt LD, Saltzman CL, SooHoo NF, Hunt KJ. Validation of PROMIS $®$ Physical Function computerized adaptive tests for orthopaedic foot and ankle outcome research. Clin Orthop Relat Res. 2013;471(11):3466-3474.

15. Hunt KJ, Alexander I, Baumhauer J, et al. The Orthopaedic Foot and Ankle Outcomes Research (OFAR) network: feasibility of a multicenter network for patient outcomes assessment in foot and ankle. Foot Ankle Int 2014;35(9).
16. Jeyaseelan L, Si-Hyeong Park S, Al-Rumaih $\mathrm{H}$, et al. Outcomes following total ankle arthroplasty: a review of the registry data and current literature. Orthop Clin North Am. 2019;50(4):539-548.

17. Jones C, Rush S, Berlet GC, et al. Understanding the postoperative course and rehabilitation protocol for total ankle arthroplasty. Foot Ankle Spec. 2015;8(3):203-208.

18. Kim HJ, Suh DH, Yang JH, et al. Total ankle arthroplasty versus ankle arthrodesis for the treatment of end-stage ankle arthritis: a meta-analysis of comparative studies. Int Orthop. 2017;41(1):101-109.

19. Kotela A, Wilk-Frańczuk M, Jaczewska J, et al. Perioperative physiotherapy for total ankle replacement in patients with inherited bleeding disorders: outline of an algorithm. Med Sci Monit. 2017;23:498-504.

20. Law TY, Sabeh KG, Rosas S, Hubbard Z, Altajar S, Roche MW. Trends in total ankle arthroplasty and revisions in the Medicare database. Ann Transl Med. 2018;6(7).

21. Lawton CD, Butler BA, Dekker RGn, Prescott A, Kadakia A. Total ankle arthroplasty versus ankle arthrodesis - a comparison of outcomes over the last decade. J Orthop Surg Res. 2017;12(1).

22. Masaracchio M, Hanney WJ, Liu X, Kolber M, Kirker K. Timing of rehabilitation on length of stay and cost in patients with hip or knee joint arthroplasty: A systematic review with meta-analysis. PLoS One. 2017;12(6).

23. McConnell EP, Queen RM. Correlation of physical performance and patient-reported outcomes following total ankle arthroplasty. Foot Ankle Int. 2017;38(2):115-123.

24. Nixon DC, Cusworth B.M, McCormick JJ, Johnson JE, Klein SE. Patient-reported allergies do not predict poorer PROMIS function, pain, and depression scores following foot and ankle surgery. Foot Ankle Int. 2018;39(8):949-953.

25. Norvell DC, Ledoux WR, Shofer JB, et al. Effectiveness and safety of ankle arthrodesis versus arthroplasty: a prospective multicenter study. J Bone Joint Surg Am. 2019;101(16): 1485-1494.

26. Oliver SM, Coetzee JC, Nilsson LJ, et al. Early patient satisfaction results on a modern generation fixed-bearing total ankle arthroplasty. Foot Ankle Int. 2016;37(9):938-943.

27. Queen R. Directing clinical care using lower extremity biomechanics in patients with ankle osteoarthritis and ankle arthroplasty. J Orthop Res. 2017;35(11):2345-2355.

28. Queen RM, Sparling TL, Butler RJ, et al. Patient-reported outcomes, function, and gait mechanics after fixed and mobile-bearing total ankle replacement. J Bone Joint Surg Am. 2014;96(12):987-993.

29. Raikin SM, Rasouli MR, Espandar R, Maltenfort MG. Trends in treatment of advanced ankle arthropathy by total ankle replacement or ankle fusion. Foot Ankle Int. 2014;35(3): 216-224.

30. Rajapakshe S, Sutherland JM, Wing K, et al. Health and quality of life outcomes among patients undergoing surgery for end-stage ankle arthritis. Foot Ankle Int. 2019;40(10): 1129-1139.

31. Rybalko D, Schwarzman G, Moretti V. Recent national trends and outcomes in total ankle arthroplasty in the United States. J Foot Ankle Surg. 2018;57(6):1092-1095. 
32. Schafer JL, Graham JW. Missing data: our view of the state of the art. Psychol Methods. 2002;7(2):147-177.

33. Schipper ON, Denduluri SK, Zhou Y, Haddad SL. Effect of obesity on total ankle arthroplasty outcomes. Foot Ankle Int. 2016;37(1):1-7.

34. Schweitzer KM, Adams SB, Viens NA, et al. Early prospective clinical results of a modern fixed-bearing total ankle arthroplasty. J Bone Joint Surg Am. 2013;95(11): 1002-1011.

35. Sutherland JM, Rajapakshe S, Younger A, et al. Do preoperative patient-reported outcomes predict hospital length of stay for surgically-treated end-stage ankle osteoarthritis patients? Foot Ankle Surg. 2020;26(2):175-180.

36. Tucker CA, Escorpizo R, Cieza A, et al. Mapping the content of the Patient-Reported Outcomes Measurement Information System (PROMIS ${ }^{\circledR}$ ) using the International Classification of Functioning, Health and Disability. Qual Life Res. 2014;23(9): 2431-2438.

37. Walter JG, Kahn SA, Noe JD, Schurman JV, Miller SA, Greenley RN. Feeling fine: anxiety and depressive symptoms in youth with established IBD. Inflamm Bowel Dis. 2016;22(2):402-408. 